\title{
La diplomacia directa como prisma de análisis de la relación UE-Cuba*
}

\author{
Direct diplomacy as an analysis prism of \\ the EU-Cuba relationship
}

\author{
Dr. Alexis Berg-Rodríguez ${ }^{* *}$
}

\begin{abstract}
RESUMEN
La firma del Acuerdo Diálogo Político y Cooperación Unión Europea-Cuba de 2016 marcó un hito en la escena internacional. El artículo analiza, desde el enfoque institucional-histórico y empírico, cómo la diplomacia estructural de la Unión y el diálogo político con Cuba crearon en 2016 el "Nuevo Modelo Político UE-Cuba”. Al analizar las entrevistas realizadas a funcionarios de la Unión, el artículo demuestra cómo la diplomacia estructural y el diálogo político en igualdad de condiciones han resultado ser más efectivos que la diplomacia tradicional y que la injerencia de la Unión en su relación con Cuba. Y cómo la diplomacia directa puede ser replicada en la relación de la Unión con Latinoamérica en el marco del Brexit.
\end{abstract}

Palabras clave: Diplomacia Directa o Estructural - Nuevo Modelo Político UE-Cuba - Acuerdo Diálogo Político y Cooperación UE-Cuba - Brexit.

* Doctor en Ciencias Políticas y de la Administración y las Relaciones Internacionales. Universidad Complutense de Madrid, 2018. Investigador visitante en el Centro de Estudios Políticos y Constitucionales en Madrid, y Profesor - Investigador en la Universitat Oberta de Catalunya, Barcelona, España. ORCID: https://orcid.org/0000-0001-8374-2568. Correo: aberg@uoc.edu. Recibido el 12 de julio de 2019. Actualizado el 6 de febrero de 2020. Aprobado el 30 de marzo de 2020 . 


\begin{abstract}
The signature of the Political Dialogue and Cooperation Agreement between the European Union and Cuba in 2016 was a landmark in the international stage. This paper examines, from the institutional-historical viewpoint and the empirical method, the way in which the use of the EU's structural diplomacy and of the political dialogue with Cuba created the New EU-Cuba Political Model, in 2016. By analyzing the data collected in interviews with EU bureaucrats, the paper shows how structural diplomacy and political dialogue on an equal basis have proven to be more efficient than traditional diplomacy and interference in the EU's relation with Cuba. Likewise, it shows that direct diplomacy can be replicated in the relationship of the EU with Latin America, in the framework of the Brexit.
\end{abstract}

Keywords: Direct or Structural Diplomacy - New EU-Cuba Political Model - Political Dialogue and Cooperation Agreement EU-Cuba - Brexit.

\title{
I. INTRODUCCIÓN
}

En este trabajo nos proponemos mostrar una perspectiva diferente en la relación Unión Europea-Cuba (UE-Cuba).Queremos subrayar cómo la diplomacia directa es mucho mejor que la condicionalidad y que la injerencia implementada por los Estados miembros de la UE a través de dos mecanismos centrales. El primero mediante la aprobación de la Posición Común contra Cuba, en 1996; mientras que el segundo elemento se realiza a través de las condicionalidades ejecutadas por la diplomacia tradicional de los Estados Miembros, siguiendo la línea establecida en la política exterior que mantiene los Estados Unidos contra Cuba a través del bloqueo económico (Berg-Rodríguez, 2019b, p. 585-597).

Por esta razón, la implementación desde 2008 de un diálogo político institucional innovador en su método, apuesta por desplazar la relación UE-Cuba del marco bilateral hacia el marco comunitario. Este desplazamiento ha permitido establecer un diálogo político en igualdad de condiciones, sin injerencia en los asuntos internos de los Estados y poniendo en el centro de la relación el bienestar de la sociedad cubana, lo que ha estimulado el cambio institucional europeo y cubano, facilitando la construcción de un Nuevo Modelo Político de relación Unión Europea-Cuba (NMP UE-Cuba). 
A este elemento se suma la implementación de la diplomacia directa de la Unión Europea y de la Alta Representante (AR), Federica Mogherini, en su relación con el Gobierno cubano, lo que ha reforzado y consolidado el NMP UE-Cuba, en tiempos en que se han producido cambios en la configuración de los Estados miembros de la UE. Además, se ha producido un cambio institucional UE-Cuba, ya que por lo general la relación de la UE con Cuba estaba subordinada a la relación que mantiene los Estados Unidos (EE.UU.) con Cuba y con el resto de los países de América Latina y el Caribe.

Este proceso se encuadra dentro del enfoque histórico-institucional de las relaciones internacionales. Dicho enfoque ha permitido identificar el "juego y el cambio institucional que realizan los actores políticos de acuerdo con sus intereses y al contexto internacional e histórico" (Vargas, 2005, p.13). Al mismo tiempo, nos permite apreciar cómo los EE.UU. mantienen desde 1960 hasta febrero del 2020 un bloqueo económico contra la sociedad y el gobierno cubano.

De hecho, lo anterior forma parte de su política doméstica, la cual fue amplificada en la relación que implementó la UE con Cuba a través de la Posición Común (PC) —desde 1996 hasta que fue suspendida en 2008Mediante la PC, la Unión y los Estados miembros condicionaron la Ayuda Oficial al Desarrollo y un aumento de la cooperación comercial, a cambio de que el Gobierno cubano realizara una transición pacífica en la isla e implementara los derechos humanos en la sociedad cubana. Para Thomas (1996, p. 91), a los EE.UU. "le interesaba que un actor internacional de peso en las relaciones internacionales, como era la UE, siguiese una política de presión y de endurecimiento hacia Cuba". Por esta razón, estas pretensiones fueron rechazadas por el gobierno y la sociedad cubana.

En este escenario, la construcción del NMP UE-Cuba, a través de la diplomacia estructural de la UE y el diálogo incondicional con el Gobierno cubano, puso de manifiesto la fragilidad y la irrelevancia de la diplomacia clásica; fundamentalmente, la implementada por los Estados Unidos contra Cuba y por los Estados miembros de la Unión, que en un primer momento se negaron a aceptar el cambio de política exterior de la UE con Cuba. De hecho, para Kennan (1951), de acuerdo con el pensamiento norteamericano, es poco plausible que los pueblos tengan aspiraciones positivas, que ellos consideren legítimas $\mathrm{y}$ a las cuales den mayor importancia que a la tranquilidad y al orden que deben regir la vida internacional. En esta línea se mantiene el gobierno de los EE.UU., mientras que los Estados miembros de la Unión consideraban insuficientes la apertura y los cambios económicos realizados por el Gobierno cubano para garantizar el bienestar 
de su sociedad y la supervivencia del sistema socialista en el siglo xxI.

Por otra parte, en la declaración realizada por Aldecoa (2018), la diplomacia directa de la UE ha logrado cortar la posible contaminación del Brexit dentro de su territorio, estimulando una mayor unidad de los Estados miembros y de los ciudadanos para profundizar en la construcción de una Unión Europea más social e independiente ${ }^{1}$. Además, estableció límites efectivos para presionar y facilitar la salida del Reino Unido de la Unión, incluido el posible retorno o no salida del Reino Unido e Irlandia, bajo la premisa de que son necesarios, pero no imprescindibles en la construcción de Europa.

En opinión de Vilariño (2000, p. 297), "se puede entender por diplomacia directa aquella que se lleva a cabo por los órganos centrales para las relaciones exteriores de un sujeto de derecho internacional, ante sus homónimos de otro u otros sujetos de derecho internacional. (...) a que a esos órganos centrales les corresponde, en principio, individual o conjuntamente, la elaboración y dirección de la política exterior del sujeto, de modo que esta se ejecuta, a través de medios diplomáticos, directamente por quienes, de un modo u otro, participan en su decisión”.

1 Declaración realizada en agosto del 2018: Curso de Verano "2019 Brexit y elecciones europeas. Una agenda crucial para el impulso de la UE”. Universidad Internacional Menéndez Pelayo. Santander. España.
Los elementos planteados hacen que el artículo se apoye en el concepto antes mencionado para abordar la diplomacia directa en el marco de la relación UE-Cuba.

Estos aspectos provocaron que la diplomacia de la UE dejara en un segundo plano la diplomacia de los Estados Unidos, ya que - en términos geopolíticos e históricos- esta ha fracasado porque ha sido incapaz de frenar la presencia de los países europeos en Cuba. En 1809, el entonces Presidente norteamericano, James Madison, consideraba que "la posición de Cuba da a los Estados Unidos un profundo interés en el destino" (Roig,1959, p. 30). Por desgracia, este posicionamiento no ha cambiado y se ha mantenido intacto por las autoridades de los EE.UU. hasta el presente siglo xxI.

En este escenario, el cambio en la relación de la UE con Cuba es resultado del nuevo orden internacional creado tras la desaparición del campo socialista. Slater (2004) considera que la existencia de categorías geopolíticas (...) tiene como objetivo la delimitación política y geográfica del espacio internacional de acuerdo a los parámetros establecidos por los poderosos. Este criterio, propio del enfoque postcolonial, deja fuera el desarrollo de un diálogo político y de la cooperación, sin previa condicionalidad y sin injerencia en los asuntos internos de cualquier país del Tercer Mundo o del Sur.

El posicionamiento de Slater no fue seguido por las partes y debido a la implementación de la diplomacia 
directa de la UE, a la puesta en marcha del NMP UE-Cuba y al diálogo político en igualdad de condiciones, han llegado a Cuba las principales instituciones europeas. Por esta razón, se puede establecer una relación de igualdad y respeto mutuo con los 28 o 27 Estados miembros, incluyendo los que forman parte del mercado común europeo.

Posiblemente, la propia UE no es consciente del enorme logro que ha alcanzado a través de la diplomacia directa ejecutada por la AR Mogherini en su relación con el Gobierno cubano. Además, puede ser replicada en su relación con el Gobierno de Venezuela, a través de un diálogo político que permita poner en el centro de la negociación el bienestar de la sociedad venezolana y despejar los intereses de terceros Estados. Para analizar esta cuestión se hace necesario partir de sus hitos y hechos.

Por esta razón, el artículo analiza la relación diplomática UE-Cuba, partiendo del examen de lo que ha sido la implementación y ejecución de la diplomacia directa por la UE en su relación con Cuba y por la AR
Mogherini en la negociación y aplicación del NMP UE-Cuba. Para contrastar nuestro estudio, analizamos esta relación con la diplomacia clásica. En segundo lugar, estudiamos los efectos de la diplomacia bilateral de los Estados miembros en su relación con Cuba, antes y en el marco del NMP UE-Cuba, especialmente la implementada por el Reino Unido, tras el triunfo del Brexit el 23 de junio del 2016.

En tercer lugar, el artículo estudia el papel que tiene la Embajada de la UE en Cuba, en su relación con el gobierno para contrarrestar el Brexit fuera de las fronteras de la Unión, como máximo representante de la diplomacia directa de la Unión y de la AR ante el Gobierno cubano. Para concluir demostrando que la diplomacia estructural y el diálogo político en igualdad de condiciones se han revelado más efectivos que la diplomacia tradicional y que la injerencia de la Unión en su relación con Cuba. Además, abre la puerta para que la diplomacia directa pueda ser replicada en la relación de la Unión con Latinoamérica.

\section{La diplomacia diRecta de la UE en Su relación con Cuba EN EL AÑO DEL BREXIT}

Los logros de la diplomacia directa de la UE se han materializado a través del NMP de relación UE-Cuba, y con la derogación de la Posición Común de la UE el 6 de diciembre de 2016, facilitando la firma del primer Acuerdo de Diálogo Político y de Cooperación (ADPC) UE-Cuba el 12 de diciembre de ese mismo año. De hecho, la diplomacia UE-Cuba se continuó reforzando con el anuncio del comienzo de la aplicación provisional del ADPC 
UE-Cuba el 1 noviembre de 2017 y con su puesta en marcha, a través de la primera reunión del Consejo Conjunto UE-Cuba el 15 de mayo de 2018, junto con la celebración en el mes de noviembre de 2018 de la primera reunión del Subcomité de Cooperación en La Habana y el diálogo político sobre medidas coercitivas unilaterales en Bruselas.

El aumento progresivo de esta relación se debe al desarrollo que ha tenido la Diplomacia Común Europea desde la entrada en vigor del Tratado de Lisboa, en diciembre de 2009, ya que supuso un gran impulso para la política exterior y para la diplomacia directa de la Unión, frente a la diplomacia tradicional de los Estados miembros. Como parte de la evolución de la política exterior de la UE, las nuevas funciones transferidas al AR por el Tratado han sido cualitativamente superiores al AR anterior, al tener nuevos cargos configurados en un paraguas de tres picos, en el que tiene como eje central y brazo ejecutor al Servicio Europeo de Acción Exterior (SEAE). En los artículos 18. 2, 3, 4 y 27.1, 2, 3 del Tratado de la Unión Europea, se establecen las funciones del AR:

1. En el art. 18.2 y 27.2 se establece que la AR es la responsable de la Política Exterior y de Seguridad Común (PESC) de la Unión Europea y de la Política Común de Seguridad y Defensa (PCSD) de Unión. En ambos casos, la AR representa a la Unión ante terceros, y está a cargo del desarrollo, planificación y ejecución de la PESC y de la PCSD de la UE.

2. Los art. 18.3 y 27.1, ponen fin a la presidencia rotatoria en el Consejo de Ministros; en este caso la AR es la presidenta del Consejo de Asuntos Exteriores. En este punto, son los especialistas y funcionarios del SEAE los directores de áreas, y los que presiden la reunión de todos los embajadores de los Estados miembros de la Unión Europea, así como las reuniones de los embajadores del SEAE, lo cual será explicado en los siguientes apartados.

3. En los art. 18.4 y 27.3 se instaura que sea la AR, Vicepresidente de la Comisión encargada de velar por la coherencia de la acción exterior de la Unión. Para el desarrollo de sus funciones, la AR cuenta con el SEAE como el instrumento que permitirá imprimir mayor coherencia y unidad a la Diplomacia Común Europea (DCE) y las embajadas de los Estados miembros.

Las funciones antes mencionadas constituyen uno de los principales avances implementados con la entrada en vigor del Tratado de Lisboa, en la esfera de la política exterior y de la diplomacia directa de la Unión Europea.

Tener a la AR de manera constante al frente de los Asuntos Exteriores de la Unión, encargada de analizar, coordinar y dar coherencia a la diplomacia común europea y responder ante las problemáticas e intereses de la UE en 
materia de PESC, permitió elevar la calidad del análisis y de la respuesta de la diplomacia directa ejecutada por la AR en su relación con Cuba.

De hecho, se ha convertido en una herramienta eficaz que ha permitido a la UE hacer frente, en la escena internacional, al Brexit británico y a las presiones del Presidente de los EE.UU., Donald Trump. De hecho, ha allanado el camino para que la UE pueda elaborar estrategias comunes por zona geográfica e imprimir así una mayor comunitarización, efectividad y coordinación entre la diplomacia de la Unión y la diplomacia tradicional de los Estados miembros. Por esta razón, la firma y aplicación provisional del NMP UE-Cuba y la gestión del Brexit por la UE habrían sido imposibles en el marco de la diplomacia tradicional, dadas las características de esta.

\subsection{La diferencia entre la llamada "diplomacia tradicional" con la "diplomacia directa o estructural"}

En sentido general, la diplomacia tradicional se basa en el Estado y concibe la actividad de forma "westfaliana", pragmática y realista - donde primarían asuntos propios como guerra, paz, disputas territoriales, comercio etc...—; la "nueva diplomacia" se centra en problemas medioambientales, derechos humanos e injerencias humanitarias. Más específicamente, la "diplomacia estructural" puede definirse como "the process of dialogue and negotiation by which actors in the
International system seek to influence or shape sustainable external political, legal, economic, social and security structure at different relevant levels in a given Geographic scene" (Moomaw, 2007; Keukeleire, at 2009; Priego, 2011).

Para Vilariño (2000, p. 314) la diplomacia directa puede desembocar en la adopción rápida y directa de resoluciones o de acuerdos susceptibles de aplicación inmediata; puede ser útil, también, para llegar a un amplio acuerdo de principios para posteriores negociaciones por misiones diplomáticas stricto sensu y para salir de un impasse en negociaciones que estén llevando a cabo estas misiones o para dar, en general, un impulso político a cualquier asunto.

Aldecoa (2011, p.152) considera que de este precepto se desprenden tres ideas claras: la primera, que el servicio diplomático trabajará en colaboración con los servicios diplomáticos de los Estados miembros; la segunda, que estará compuesto por funcionarios de la Secretaría de los servicios diplomáticos nacionales, y la tercera, que la organización y el funcionamiento del SEAE será establecido mediante una Decisión del Consejo.

Estos elementos han facilitado la firma y aplicación provisional del Acuerdo UE-Cuba. Al mismo tiempo, han permitido que el alcance del primer ADPC UE-Cuba tenga una transcendental relevancia internacional, institucional y diplomática para ambas partes. De hecho, presenta una gran importancia en la esfera de la 
diplomacia, por ser un triunfo de la Diplomacia Directa implementada por la UE y la Alta Representante Federica Mogherini, durante el proceso de negociación del Acuerdo con el Gobierno Cubano. A este proceso se suma el apoyo de la diplomacia latinoamericana, alentando a la Unión Europea, en las Cumbres UE-Comunidad de Estados Latinoamericanos y Caribeños (CELAC) de 2013 y 2015, a comenzar la negociación y firmar el Acuerdo con Cuba.

De la misma forma, en los encuentros ministeriales de asuntos exteriores UE-CELAC 2016 y 2018, la AR Mogherini certificó la necesidad de reforzar su relación multilateral recogida en la Estrategia Global de la UE de 2016, y que ha sido implementada a través de la diplomacia y la cooperación internacional para alcanzar los objetivos comunes y de interés global. "Cuando unimos nuestras fuerzas podemos ser una fuerza global en favor de causas positivas. No habría Acuerdo de París sobre el Cambio Climático, ni Objetivos de Desarrollo Sostenible, sin el trabajo diplomático conjunto que llevamos a cabo durante meses y años" (Mogherini, 2016, p. 13).

En este escenario, los pasos dados por ambos bloques regionales, a través de la diplomacia directa, han reforzado la relación UE-Cuba a través del NMP y entre la UE con los países latinoamericanos a través de la negociación de diferentes acuerdos de cooperación e intercambios comerciales con esta región.
En este escenario, la AR Mogherini logró implementar, progresivamente y de común acuerdo con el Gobierno cubano, la diplomacia directa de la UE, en el proceso de negociación del NMP. Con este paso, ambas partes garantizaron el éxito de la negociación, ya que era "un tema muy sensible y de máxima importancia para las autoridades cubanas y europeas". Esta dimensión del NMP UE-Cuba ha quedado plasmada en las entrevistas que fueron realizadas a los especialistas ${ }^{2} \mathrm{del}$ Servicio Europeo de Acción Exterior (SEAE), que integraron la delegación de la UE para realizar la negociación del Acuerdo de Cooperación con Cuba y en cada una de las visitas oficiales que realizó la AR Federica Mogherini a Cuba para alcanzar la cristalización y puesta en marcha del NMP UE-Cuba.

\subsection{La implementación de la} diplomacia directa en el contenido y funcionamiento del NMP UE-

\section{Cuba}

El trabajo de la AR Mogherini en 2016 estuvo marcado por la celebración del referéndum sobre el Brexit en el Reino Unido y por la presentación al Consejo Europeo de la Estrategia Global para la Política Exterior y de Seguridad (egPes) "Una visión común, una actuación conjunta: una Europa más

2 N. del A: En la actualidad los funcionarios entrevistados forman parte del equipo del SEAE encargado de poner en marcha el Acuerdo. Por esta razón, no se brinda la información sobre sus nombres y cargos. 
fuerte". De hecho, la nueva Estrategia Global de Seguridad incorpora un "enfoque global y de acción conjunta" ${ }^{33}$ la política exterior y de seguridad de la UE, rasgo que no tenía la anterior Estrategia de Seguridad.

La actual estrategia de la AR Mogherini no presenta una visión abstracta de la UE, al estar abierta a los intereses comunes de los Estados miembros y al ejercicio práctico de la diplomacia común europea, con la intención de alcanzar una actuación conjunta entre la diplomacia de los Estados miembros y la diplomacia común de la UE, que permita la construcción de una Europa más fuerte y unida. Por esta razón, la UE necesita una diplomacia común europea, "que promueva nuestros intereses comunes mediante un compromiso responsable y la cooperación de los demás” (Mogherini, 2016, p. 41).

Si analizamos que la fecha de elaboración y de presentación de la Estrategia Global de la AR Mogherini transcurre entre los años 2014 y 2016, se puede observar cómo se ha diseñado una Estrategia Global ajustada a la realidad internacional y regional con una visión de futuro, que ha permitido a la UE asumir los nuevos retos en el corto, mediano y largo plazo. En este período se enmarca el repunte de los ataques terroristas en la UE, el

3 "Global" no debe entenderse únicamente en el sentido geográfico: también hace referencia al amplio espectro de políticas e instrumentos que promueve la estrategia. acercamiento de los EE.UU. y Cuba bajo el gobierno de Barack Obama, la Cumbre de París, las negociaciones y firma del primer adpc UE-Cuba, el Brexit, las elecciones en Francia y los EE.UU., entre otros acontecimientos de impacto global.

Esta visión global fue aplicada por la diplomacia de la UE en el contenido que dio vida al NMP a través del ADPC UE-Cuba de 2016. En su estructura, las partes ajustan el Acuerdo al contexto regional e internacional histórico en la que se estaba gestando el NMP UE-Cuba y que quedó marcada por la aprobación del Brexit por parte de la sociedad del Reino Unido. Por esta razón, se recoge en único Tomando Nota del Acuerdo la posibilidad que tienen el Reino Unido e Irlanda para "adherirse a acuerdos específicos en el ámbito de la libertad, la seguridad y la justicia,celebrados por la Unión Europea" y que forman parte del NMP. La UE y Cuba han dado este paso, ante la posible salida o no del Reino Unido de la UE y a la especial relación que puede tener el Reino Unido con Irlanda.

En el Acuerdo las partes han recogido los valores y principios con los que se identifican y que intentan exportar de manera directa a sus sociedades y a la sociedad internacional en una relación en la que prima el desarrollo del diálogo político para garantizar el bienestar de la sociedad cubana, reforzando la cooperación y el comercio. Este objetivo debe permitir a la UE y Cuba dar respuestas comunes a retos globales, como el medio ambiente y 
la migración, y promover la comprensión mediante el fomento, a todos los niveles, de los contactos, el diálogo y la cooperación entre la sociedad cubana y las de los países de la Unión Europea. Por otra parte, los intereses de los Estados miembros en la negociación del ADPC UE-Cuba hizo que el Acuerdo con Cuba se convirtiera en uno mixto, respondiendo al interés de los Estados miembros. En este escenario, y para evitar que los intereses de terceros países y de los Estados miembros, incluyendo al Reino Unido, pudieran interferir en los intereses comunes de las partes y en el correcto funcionamiento del nmp UE-Cuba. La UE y Cuba establecieron como parte de la estructura del ADPC la creación del Consejo Conjunto como órgano encargado de aplicar y supervisar las decisiones que se tomen en sus reuniones.

De hecho, las decisiones que se tomen son de carácter vinculante para las partes y previamente negociadas a través de la realización de un Diálogo Político que abarca las esferas a negociar. Los Estados miembros van a participan como observadores en todos los artículos que no sean de su competencia, cuando sea ratificado el acuerdo, y solo pueden hacer recomendaciones. Esta decisión va a tener presente que la propuesta responda a los intereses de las sociedades cubanas-europeas y que no sea contraria a los intereses comunes de las partes.

Por esta razón, el diálogo político es el eje central del Acuerdo y el puente para garantizar que cada una de las partes ejecute el Acuerdo, ya que siempre va a responder a sus intereses comunes, sin ningún tipo de injerencia. Otro elemento relevante que acompaña el Acuerdo UE-Cuba es que no existe un período determinado para efectuar la ratificación del Acuerdo por parte de los Estados miembros ni en las decisiones del Consejo. Hasta el 5 de febrero de 2020 solo faltan 2 Estados miembros por ratificar el ADPC con Cuba.

Tampoco tenemos una fecha límite para ratificar los acuerdos y no sabemos cuándo sería, porque depende de los parlamentos de cada Estado miembro, pero de 2 a 3 años debería ser lo máximo. Esta situación no nos preocupa, porque se va a aplicar aproximadamente el $98 \%$ del Acuerdo (Entrevista con especialista del SEAE vía telefónica en Bruselas-Madrid 20 de noviembre de 2017).

El Acuerdo Mixto UE-Cuba puede aplicarse provisionalmente y de manera parcial en las competencias que son de aplicación exclusiva de la Unión y abarcan 83 artículos de los 89 que conforman el ADPC. En este escenario, la aplicación provisional de los 83 artículos del ADPC UE-Cuba se pudo lograr gracias a la diplomacia directa y al Diálogo Político realizado por las partes.

\subsection{La tercera visita de la $A R$} Mogherini a Cuba: reforzando el diálogo y la diplomacia directa

En el contexto antes mencionado, la visita realizada en el mes de enero de 
2018 tenía como principal objetivo reforzar el nuevo modelo político de relación UE-Cuba, a través del diálogo político y de la cooperación, en un momento de retroceso en la relación de Cuba con los EE.UU.

"La diplomacia directa no es, sin embargo, una forma moderna de diplomacia, pues desde los tiempos más remotos, al menos ya en la Antigüedad documentada, los responsables de los entes políticos, los príncipes medievales o los soberanos absolutos, o los más modernos responsables del gobierno de los Estados, no han dejado de entrevistarse (...) Tampoco es moderna por la técnica que utiliza, que es la tradicional de la negociación confidencial, reservada o secreta" (Vilariño, 2000, p. 305-306).

Como principal resultado destaca el apoyo institucional de la UE al Gobierno y a la sociedad cubana: "en este momento hay quienes intentan aislar a Cuba. Nosotros, los europeos, queremos mostrar, por el contrario, (...) Frente a los que levantan muros y cierran puertas, nosotros los europeos queremos tender puentes y abrir puertas mediante la cooperación y el diálogo" (Mogherini, 2018, p. 2).

El respaldo realizado por la AR forma parte de la diplomacia directa de la UE y terminó estimulando el aumento de las relaciones diplomáticas entre ambas partes, respaldado por las tres visitas realizadas por la AR Federica Mogherini a Cuba desde que ocupó su cargo a finales de 2014 hasta el verano de 2019. En este período, la AR
Mogherini se puso al frente de la implementación provisional del Acuerdo ante los grupos parlamentarios contrarios a la firma y al comienzo de la aplicación provisional del ADPC UE-Cuba.

La tercera visita de la AR Mogherini a Cuba, en enero de 2018, ha servido para poner en marcha la Comisión Conjunta del ADPC, tras recibir el respaldo del Presidente Raúl Castro, junto con el respaldo de quien sería, unos meses más tarde Presidente de Cuba, Miguel Díaz-Canel, y del actual Ministro de Asuntos Exteriores, Bruno Rodríguez, para poner en marcha el Acuerdo y, con él, un nuevo modelo político-institucional-jurídico entre las partes.

"[M]ientras EE.UU. ha optado
por el aislamiento para provo-
car la caída del régimen castrista,
Bruselas ha matizado su línea
de actuación por medio de ges-
tos políticos en busca de una di-
plomacia pragmática, cuya ma-
nifestación más evidente se ha
materializado con el cierre de
las negociaciones del Acuerdo
de Diálogo Político y Coopera-
ción, en marzo de 2016” (Ortiz,
2016, p. 339).

A través de la diplomacia directa, la AR Mogherini ha facilitado que la UE muestre su apoyo a los cambios estructurados por el Gobierno y la sociedad cubana, en el plano político, social y económico. Mientras que, por otra parte, permite que la UE tenga una 
mayor visibilidad y posicionamiento en América Latina, dada la importancia simbólica y moral que tiene Cuba en la región. Por ser el único país que derrotó - en 1961-a los Estados Unidos, en la invasión de playa Girón (Bahía de Cochino) y resistió el bloqueo norteamericano durante más de cinco décadas. De hecho, la diplomacia directa ha permitido que la UE (2017, p. 4) pueda implementar, junto con Cuba, varios proyectos de cooperación por un valor total de 330 millones de euros entre 2008 y 2020, con un impacto directo en la sociedad cubana.

Por otra parte, la implementación de la diplomacia estructural de la UE en su nueva relación con Cuba facilitó que el NMP de relación UE-Cuba se pudiera construir e implementar a través del diálogo político institucional y diplomático, permitiendo a las partes reforzar su colaboración en la esfera de la cooperación y el comercio, en igualdad de condiciones y eliminando todo tipo de injerencia en su relación, creando un espacio de confianza mutua entre las partes.

La diplomacia directa de la AR Mogherini estimuló la celebración de varios diálogos políticos sobre Derechos Humanos UE-Cuba, siendo el resultado de la cohesión y coordinación realizada por la AR, el Gobierno cubano y el SEAE. Como consecuencia de lo anterior, Cuba ha logrado negociar y poner en marcha el ADPC con la UE sin ceder su soberanía y sin cambiar su sistema político, en un ambiente de confianza mutua y sin ningún tipo de presión entre las partes.

Con la firma del Acuerdo se crea un marco para el diálogo político aún más fuerte, para nuevos diálogos sobre sectores específicos y para ampliar y concretizar la cooperación en una gran variedad de temas, considerando que, para la Unión, el Acuerdo de Diálogo Político y de Cooperación proporciona un instrumento para apoyar mejor el proceso cubano de modernización económica y social (Mogherini, 2016).

Por esta razón, la estrategia seguida por la AR para garantizar la implementación del ADPC provisionalmente supuso que la Unión Europea aprobara un presupuesto para la Ayuda Oficial al Desarrollo (AOD) ascendente a 50 millones de euros para los años 2014 a 2020. Para alcanzar el mismo objetivo, se realizó la primera reunión del Subcomité de Cooperación en la Habana con el objetivo de garantizar el presupuesto de AOD para Cuba en el período 2021-2027. Para seguir reforzando la relación institucional UE-Cuba, en el marco del Foro de Negocios de Cuba en La Habana, en junio del 2019, visitó la isla el Comisario Europeo para la Cooperación Internacional y el Desarrollo, Neven Mimica.

Este posicionamiento es la clave y el eje central de la diplomacia estructural de la UE y sobre él hay que hacer girar la nueva relación UE-Cuba, respaldando la Unión las diferentes transformaciones realizadas por el 
Gobierno cubano, de manera progresiva, sin acelerar sus tiempos y las pautas marcadas por este.

En el proceso, la diplomacia directa de la UE estuvo respaldada por el diálogo y por las diferentes acciones diplomáticas realizadas por el Gobierno cubano. Estas acciones no dejaron margen a la duda a la sociedad internacional de que los cambios en su política interior y exterior eran para garantizar el bienestar de la sociedad cubana y la supervivencia del sistema socialista cubano en el siglo xxI. En este caso, destaca la negociación que hizo La Habana para renegociar la deuda con el Club de París y con el Gobierno de España.

De hecho, las medidas recogidas en el artículo 10 del ADPC y en el diálogo político sobre las medidas coercitivas unilaterales puesto en marcha en noviembre de 2018, no frenan las sanciones ni las medidas que los Títulos III y IV de la Ley Helms-Burton de los Estados Unidos aplica desde mayo de 2019 contra las empresas europeas que comercializan con Cuba. Este espacio puede facilitar que las partes creen las herramientas que permitan garantizar una eficaz implementación del Acuerdo en beneficio de la sociedad cubana, europea y mundial, frenando las sanciones y las subidas arancelarias de los Estados Unidos.

Con este paso, la UE puede continuar dando pasos para alcanzar una mayor autonomía respecto de la política exterior de los EE.UU. Para contrarrestar las sanciones del bloqueo, la UE puede implementar una batería de contramedidas contra las empresas e instituciones norteamericanas. En opinión de Berg-Rodríguez (2019b, 585-597) esta posible reacción puede activar el diálogo político que la UE y los EE.UU. estaba realizando desde 1996 para frenar las sanciones que sigue imponiendo el Departamento del Tesoro norteamericano a las empresas europeas que están comercializando con el gobierno y la sociedad cubana.

Esta realidad hace que 23 años después del comienzo de la Cumbre de Londres UE-Estados Unidos, se mantenga el carácter extraterritorial del bloqueo contra Cuba y que afecte directamente a las empresas europeas. También se prevé que aumenten las sanciones y demandas después de la entrada en vigor, en mayo de 2019, del Título III y IV de la Ley Helms Burton, al autorizar que los ciudadanos norteamericanos puedan demandar a las empresas europeas y de todo el mundo que tenga negocios en Cuba (Berg-Rodríguez, 2019b, p. 585-597).

A través del análisis de estas relaciones se pone en evidencia un desplazamiento de la relevancia en la diplomacia clásica correspondiente a la esfera bilateral frente a la diplomacia directa del ámbito multilateral representado por la AR Mogherini. Este escenario se debe a que el peso de esta relación sobrepasa los aspectos formales e institucionales, al tener un peso decisivo en las negociaciones que se 
realizaron para alcanzar la firma del ADPC con Cuba. Este elemento hace que su importancia siga aumentando en el marco del NMP y en la escena internacional a través de los diferentes encuentros directos que ha realizado la AR Mogherini con el gobierno cubano y con el resto de los dirigentes de la región.

A este escenario hay que añadir al grupo de negociación propuesto por la AR para establecer un diálogo entre el gobierno venezolano y el presidente de la Asamblea de Venezuela. Con este paso, la UE trata de desterrar las posiciones de los Estados miembros, que abogaban por la injerencia y la condicionalidad como la vía de solución a la inestabilidad que ha provocado el presidente de la Asamblea de Venezuela al autoproclamarse como Presidente de la República. Esta situación brinda la oportunidad de replicar el NMP UE-Cuba en las relaciones con el Gobierno venezolano, siempre que se ponga en el centro de la relación velar por el bienestar social y económico de la sociedad venezolana frente a los intereses de terceros Estados.

\section{Efectos de la diplomacia bilateral de los Estados MIEMbROS EN SU RELACIÓN CON CUBA, EN EL MARCO DEL NMP UE-Cuba y DEL BREXIT}

En sentido general, la relación diplomática entre los Estados miembros y el Gobierno cubano ha incidido directa e indirectamente en la relación multilateral que la UE y el Gobierno cubano han tenido desde su creación. Esta situación ha sido posible por la existencia, en un primer momento, de dos ejes dentro de los Estados miembros: el eje atlántico, representado por el gobierno de España de José María Aznar, al que luego se sumó el eje del Este con la República Checa junto con Polonia a la cabeza. En ambos casos los bloques respaldaban la política de los Estados Unidos contra Cuba frente al eje europeísta, interesado en firmar un Acuerdo de Cooperación Comercial con La Habana.
Como parte del juego histórico-institucional, los Estados miembros del eje atlántico respaldaron la propuesta realizada por el presidente español al Consejo de Florencia de 1996, para que fuera aprobada la Posición Común (PC) de la UE contra Cuba, en lo que fue el primer momento crítico en la relación de la UE-Cuba, que surgió fruto de los intereses de los propios Estados miembros. Mientras que el segundo momento crítico se produjo en el marco del desarrollo del diálogo político entre las partes, que permitió la apertura de la Oficina de la Comisión Europea en La Habana y la presentación de la solicitud del Gobierno cubano para incorporarse al Acuerdo de Cotonou en 2003. 
El aumento del bloqueo de los EE.UU., contra Cuba, a través de la Ley Helms-Burton de 1996, provocó que el Gobierno cubano aplicara la Ley 88 de Protección de la Independencia Nacional y la Economía de Cuba de 1999, realizando la "detención de 75 disidentes" por responder a los intereses de los EE.UU., aplicando "la pena de muerte a tres terroristas" (Vadillo, 2011, p. 22).

La respuesta de la UE y de los Estados miembros ante esta actuación del Gobierno cubano dejó al descubierto la prepotencia y las verdaderas intenciones que tenía en su relación con Cuba, demostrando lo vinculado que estaba el discurso europeo a la política de los EE.UU. En opinión del Gobierno cubano y de varios autores, estos acontecimientos diplomáticos constituyeron una clara injerencia de la UE y de los Estados miembros en los asuntos internos de Cuba. Esta situación provocó que el Gobierno cubano se negara a seguir recibiendo AOD procedente de la UE y que retirara su solicitud para formar parte del Acuerdo de Cotonou.

Como era de esperar, La Habana rechazó esta posición de la UE por intervencionista, y además la "posición común" también se convirtió en un asunto contencioso entre los países miembros de la UE. El corsé probó ser resistente: más de una década y media después, la posición común se mantiene en su sitio. Sin embargo, su relevancia práctica se ha erosionado (Hoffmann, 2012, p. 123-124). Al mismo tiempo, fue un reflejo de las tensas relaciones diplomáticas que mantenían la UE y sus Estados miembros con Cuba, después de la caída del muro de Berlín, y justo cuando la soledad comercial y política de la isla era prácticamente total.

En este contexto, los países miembros de la UE más orientados al diálogo, impedidos de deshacer o de cambiar la posición común, se dedicaron a destacar sus relaciones bilaterales individuales con La Habana por encima de aquellas dentro del contexto de la UE (Hoffmann, 2012, p. 123-124). Por esta causa, Cuba continuó recibiendo la ayuda a la cooperación procedente de los diferentes Estados miembros del eje europeísta y de sus instituciones intergubernamentales, en el marco de la no injerencia y estimulando el restablecimiento del diálogo político con el Gobierno cubano. A este proceso se podía unir de manera intermitente o irregular algún Estado miembro del eje atlántico y del Este, dependiendo del partido que estuviera en el gobierno.

En este caso destacan la visita a Cuba del Presidente francés François Hollande, en mayo de 2015, y posteriormente la vista de Raúl Castro a Francia, en febrero del 2016, siendo ambas hechos históricos por ser las primeras visitas que los principales dirigentes de ambos países realizaban en los años de relación bilateral.

Por esta razón, ambos encuentros sirvieron para consolidar el vínculo diplomático que se venía desarrollando desde 2012, y respaldar los cambios 
realizados por el Gobierno cubano por parte del Gobierno francés. De hecho, Francia se convirtió en el país que más impulsó y lideró la negociación del Acuerdo de la UE con Cuba.

Mientras que el papel que jugó el gobierno del Partido Popular de España quedó en un segundo plano y solo se observó un mayor acercamiento por la restauración de la deuda externa que realizó el gobierno español al cubano en 2016, como una estrategia para compensar el distanciamiento durante el proceso de negociación del ADPC UE-Cuba.

En este contexto, la visita relámpago realizada por el Presidente de España, Pedro Sánchez (PSOE), en noviembre de 2018, solo alcanzó a reestablecer la relación a nivel presidencial. En todo caso, el alcance e impacto de esta visita se puede ver reforzada gracias a que Pedro Sánchez logró configurar un gobierno de izquierda, y por la visita realizada por los reyes a la Isla, el pasado 12 de noviembre del 2019.

\subsection{La diplomacia del Reino Unido con Cuba en el marco del Brexit}

En este escenario, el Reino Unido (RU) ha emprendido una veloz carrera diplomática para retomar los Acuerdos que pueden resultar más estratégicos para su país y que de una forma u otra puedan devolver la grandeza imperial del Reino Unido.

Para alcanzar este objetivo, en el mes de abril de 2016 visitó La Habana
Philip Hammond, Secretario de Estado para los Asuntos Exteriores y de la Mancomunidad del Reino Unido de Gran Bretaña e Irlanda del Norte, siendo la "primera ocasión en que un jefe de la diplomacia británica visitaba Cuba desde 1959" (Valdés, 2016), y dos meses antes de que se efectuara el referéndum del 23 de junio de 2016, era toda una declaración de intenciones, ya que la diplomacia británica utilizó el paraguas de la construcción del NMP UE-Cuba para tener este primer contacto con el gobierno cubano.

En esta oportunidad, el Secretario británico reconoció el gran potencial para seguir mejorando esos nexos en los próximos meses y años. De hecho, sostuvo que el Reino Unido puede hacer mucho más en términos de relaciones comerciales, turismo e intercambio pueblo a pueblo. A través de esta declaración, la diplomacia británica sentaba las bases para desarrollar el intercambio comercial con Cuba una vez que saliera de la UE.

De la misma manera, el Secretario de Estado dejó patente la voluntad de fortalecer el diálogo bilateral en temas de interés común y reforzar la cooperación mutua respaldada por mayores niveles de relaciones comerciales y económicas. Para fortalecer la cooperación bilateral entre ambos países, se firmaron cuatro Memorandos de Entendimiento entre los ministerios cubanos de Energía y Minas, Educación Superior, Cultura y el Banco Central de Cuba, y el ministerio de Relaciones 
Exteriores y de la Mancomunidad del Reino Unido de Gran Bretaña e Irlanda del Norte.

En 2017 el embajador Antony Stokes dejaba el verdadero mensaje que la diplomacia británica quería transmitir al gobierno cubano: que la relación con la Unión todavía está por determinarse, y que estaban montando un nuevo enfoque global para el Reino Unido, en este contexto el fortalecimiento de la cooperación con Cuba era un ejemplo de ello.

A pesar de esta proyección británica, dos años después, la relación diplomática Cuba-Reino Unido ha avanzado muy poco y no se han implementado las proyecciones iniciales ni las propuestas realizadas por el canciller británico, salvo la visita realizada a Cuba, en marzo de 2019, por el príncipe de Gales como parte del despliegue diplomático que está desarrollando el Reino Unido en el marco del Brexit.

Asimismo, no se ha materializado ningún proyecto, aunque esta estrategia puede ser considerada como un proceso de preparación del escenario internacional antes dejar la UE. Hasta que el Reino Unido pueda tener un gobierno capaz de alcanzar una eficaz vuelta a la escena internacional, se hacía imprescindible seguir dilatando la salida de la Unión y tratar de tener un buen resultado en las negociaciones con la UE.

Por mucho que el Reino Unido haya tratado de limitar la diplomacia directa de la UE, no hay una diplomacia bilateral con los Estados miembros que sea más reconocida por el gobierno cubano y que sea más efectiva que la diplomacia estructural que mantiene con la UE.

\subsection{Las Embajadas de la UE como} herramientas de la diplomacia directa: papel e importancia en el NMP y en tiempos del Brexit

La labor de los embajadores de la Unión ha facilitado la consolidación de la UE como un actor importante a nivel global y que tenga mayor visibilidad en el actual contexto internacional, a pesar de las voces que plantean lo contrario. Con la creación del Servicio Europeo de Acción Exterior por el Tratado de Lisboa y la puesta en marcha del SEAE, las delegaciones de la Comisión se han convertido en las Embajadas o Delegaciones de la UE.

A partir de su puesta en marcha, el SEAE cuenta con ciento cuarenta delegaciones en el mundo, incluyendo la oficina en Cuba, que en el año 2011 fue promovida a embajada de la UE dentro de su estructura.

En el funcionamiento de esta repartición diplomática, el embajador es el máximo responsable de aplicar cada una de las funciones recogidas en el artículo 5 de la Decisión del Consejo 427/2010, lo cual permite que la Delegación de la UE funcione como una misión diplomática similar a cualquier otra embajada acreditada en el país, al desempeñar funciones parecidas, pero 
con mayor alcance. De hecho, el Embajador de la UE tiene que representar a la Unión y presidir de manera permanente a los embajadores de los Estados miembros. También tiene que defender la posición de la Unión en el marco del NMP, así como participar en la preparación y negociación de los acuerdos entre Cuba y la UE, dando seguimiento de todos los acuerdos firmados.

Las facultades conferidas al Embajador de la UE han contribuido a que esta tenga una voz común en su relación con Cuba, facilitando — desde su puesta en marcha en 2011- el restablecimiento del diálogo político y de cooperación entre las partes. El trabajo realizado por la Delegación de la UE abrió la puerta para el inicio de la negociación del Acuerdo y el desarrollo de cuatro diálogos sobre derechos humanos, sin que exista la interferencia de otros Estados miembros. Igualmente, ha contribuido a reforzar el diálogo institucional entre la UE y el Gobierno cubano, al ser el único interlocutor válido de la UE en la Isla, permitiendo a la Unión exportar sus valores democráticos a la sociedad cubana.

Por otra parte, los embajadores de la UE son responsables ante el AR de la gestión global del trabajo que realice la delegación y de garantizar la coordinación de todas las acciones de la Unión en los terceros países (entrevista al especialista del SEAE en Santander, 27 de septiembre del 2016).

En cierto modo, esta dualidad entre Consejo y Comisión podría equivaler a la dualidad entre "diplomacia tradicional" y "estructural", lo que supone un interesante caso innovador para la práctica diplomática contemporánea. Las delegaciones apoyarán a los Estados miembros en sus relaciones diplomáticas y en la función de prestar protección consular a los ciudadanos en terceros países (Priego, 2011, p. 5).

El funcionamiento de las embajadas de la Unión está dirigido por un embajador del SEAE, que es designado por la AR, contando con las mismas protecciones consulares que los embajadores de los Estados miembros. Por otra parte, tenemos que aclarar que las reparticiones diplomáticas de la Unión Europea no son embajadas de la Comisión, ni de los Estados miembros, constituyendo este elemento uno de los principales avances que se introducen en el Tratado de Lisboa de 2009. "En mi caso, me toca coordinar mi trabajo con el embajador de Francia, Italia, Alemania y Polonia, por solo mencionar algunos (...). Al mismo tiempo, decidimos qué embajador será el más indicado para leer un comunicado o declaración de prensa en representación de la UE, siempre que sea necesario" (entrevista al especialista del SEAE en Santander, 27 de septiembre de 2016).

Por esta razón, "el SEAE constituye una de las innovaciones institucionales de mayor alcance político producidas en el seno del proceso de integración europea, desde sus orígenes hasta nuestros días" (Cornago, 2011, p. 82). Por este motivo, se considera el SEAE 
como una herramienta necesaria para el desarrollo efectivo, coordinado y coherente de la diplomacia común europea, lo cual ha permitido directamente brindar a la UE una mayor visualización y reconocimiento en la escena internacional.

A través del trabajo de las embajadas de la UE, se amplía el servicio exterior de la Unión en su conjunto y en la centralización de toda la política exterior, de seguridad y defensa común, en un solo responsable en los terceros países, hecho que posiblemente haya facilitado una mayor coordinación y coherencia entre el SEAE y el servicio exterior de los Estados miembros, al momento de implementar las políticas y acciones exteriores de la UE.

Este resultado es el fruto del trabajo realizado por la AR Federica Mogherini, que ha logrado mantener de manera coordinada, eficaz y coherente, el funcionamiento del SEAE y de la DCE con los intereses diplomáticos de los Estados miembros.

Los elementos antes planteados muestran a la Unión Europea como un actor diplomático estructural no solo por el poder normativo que caracteriza su política exterior, al incluir los diferentes temas que aborda la DCE - protección de los derechos humanos, el medio ambiente, los crímenes de lesa humanidad, el terrorismo internacional, el comercio internacional, la buena gobernanza, la paz mundial, entre otros- sino también porque ha logrado implementar con el Gobierno cubano cuatro diálogos políticos sobre Derechos Humanos, un espacio de respeto mutuo y donde la sociedad cubana y europea están en el centro de la relación UE-Cuba.

Con la entrada en funcionamiento del AR, del SEAE y de la Diplomacia Común Europea (DCE), la UE ha ganado en eficacia y efectividad en la exportación de los valores que caracterizan a la Unión. Este resultado se debe, en gran medida, a la coherencia y coordinación que imprime el AR y su brazo ejecutor en la DCE, validando su trabajo como la vía más efectiva para que la UE aborde las esferas antes mencionadas y, con ello, se convierta en líder del nuevo orden internacional.

El hecho de que las delegaciones de la UE sean las encargadas de aplicar la diplomacia directa confiere una alta responsabilidad para los embajadores de la Unión y muy especialmente para el destinado en Cuba, por cuanto es el responsable de seguir reforzando las relaciones UE-Cuba y continuar contribuyendo a estrechar las relaciones entre la sociedad cubana y europea, por ser las principales receptoras de los beneficios del Acuerdo.

Por otra parte, "las delegaciones tienen un importante papel en la política de desarrollo europeo, especialmente desde la descentralización iniciada en el año 2000. Conducen el diálogo político que va a alimentar la programación de la cooperación con los países socios y funcionan como 'ventanillas' o administración de referencia para todos los proyectos que se vayan a 
plantear o se estén ejecutando en el país" (Guinea, 2011, p. 118).

A este reto hay que sumar el desafío que tiene la UE en el siglo xxI, para lograr ser un actor global independiente en el actual contexto post hegemónico y ante el cambio de la política exterior de los Estados Unidos. En este camino, el trabajo de la AR Federica Mogherini será esencial para permitir a la UE mantener un sello de identidad propio en su PESC y en la PCSD, siempre que la UE desee promover, como líder del nuevo orden internacional, los valores y principios de los derechos humanos, la democracia, y la buena gobernanza.

\section{Conclusiones}

En este momento no existe en la UE un Estado miembro que tenga la capacidad de diseñar una política exterior con una estrategia y un servicio diplomático comparado con el servicio de acción exterior de la Unión Europea, y que permita a la misma hacer frente, de manera eficaz, coordinada y coherente, al cambio del sistema internacional y a la implementación y ejecución del Nuevo Modelo Político de relación UE-Cuba, así como a los desafíos que está generando en el escenario internacional el cambio de la política exterior de los Estados Unidos hacia América Latina y el Caribe, el aumento del bloqueo norteamericano contra Cuba, y la materialización o no del Brexit.

Tras analizar la diplomacia directa como una herramienta de la política exterior de la UE, podemos concluir que la diplomacia desarrollada por los Estados miembros en su relación bilateral con Cuba y con terceros Estados tiene menos relevancia y peso que la implementada por la AR Mogherini en representación de la UE. De hecho, la diplomacia británica en su relación con Cuba y sus antiguas colonias tiene menos peso frente a la acción que está realizando la diplomacia común europea.

Estos resultados muestran a la UE como un actor diplomático estructural no solo por el poder normativo que caracteriza su política exterior, sino por incluir dentro de la diplomacia la protección de los derechos humanos, el medio ambiente, el terrorismo internacional, la buena gobernanza y la paz mundial, entre otras.

De hecho, la diplomacia directa de la UE en su nueva relación con $\mathrm{La} \mathrm{Ha}$ bana y el diálogo político en igualdad de condiciones, facilitaron que el NMP UE-Cuba pudiera construir un espacio de respeto mutuo, poniendo en el centro de la relación el bienestar de la sociedad cubana y la supervivencia de su sistema socialista. Esta característica ha permitido a las partes reforzar su colaboración en la esfera de los derechos humanos, la cooperación y el comercio, en igualdad de condiciones 
y eliminando todo tipo de injerencia en su relación.

Por otra parte, la relación diplomática UE-Cuba siempre ha estado atravesada por la que han mantenido los Estados miembros con el Gobierno cubano y que varía de acuerdo con la empatía política e intereses económicos de los gobiernos que lleguen al poder. En estas relaciones diplomáticas no se ha construido un proyecto sostenido en el tiempo, ya que cambian de acuerdo al partido que asuma el gobierno. Bajo esta característica, nunca se hubiera podido construir el NMP UE-Cuba.

Para evitar que los intereses de los Estados Unidos y de los Estados miembros pudieran interferir en el funcionamiento del NMP UE-Cuba, se ha creado el Consejo Conjunto como órgano encargado de aplicar y supervisar las decisiones que se tomen en el marco del ADPC. Estas decisiones son de carácter vinculante para las partes y los Estados miembros participan como observadores en 83 de los artículos que configuran el ADPC.

A través del trabajo de las embajadas de la UE, esta ha logrado tener una única voz, centralizando toda la política exterior, la diplomacia directa y el diálogo político en un solo responsable en los terceros países. Esta característica ha facilitado una mayor coordinación y coherencia entre el SEAE y el servicio exterior de los Estados miembros, para implementar la política común de la UE con Cuba y los terceros países.

Se hace imprescindible replicar el NMP UE-Cuba en las relaciones y en los contactos que tiene la UE y el SEAE con el Gobierno venezolano, para garantizar que se preserve el bienestar social y económico de la sociedad venezolana frente a los intereses de terceros Estados.

\section{Bibliografía}

Agencia EFE (2019).“El heredero británico llega a Cuba en una misión de diplomacia constructiva". Recuperado el $10 \mathrm{de}$ julio de 2019 de www.efe.com/efe/america/politica/el-heredero-britanico-llega-a-cuba-en-una-mision-de-diplomacia-constructiva/20000035-3933819

Aldecoa, F. (2011). "La diplomacia europea. El servicio europeo de acción exterior", en Francisco Aldecoa Luzárraga. (Coord), (2011). La diplomacia común europea: el servicio europeo de acción exterior. Madrid: Marcial Pons, pp.152. Berg-Rodríguez, A. (2019a). La reforma constitucional en Cuba en el marco de la aplicación provisional del Acuerdo UE-Cuba. Revista Oñati Socio-Legal Series. [online], 9(6), 924950. Recuperado el 30 de enero de 2020 de https://doi.org/10.35295/osls. iis//0000-0000-0000-1109 
Berg-Rodríguez, A. (2019b). "Rol de la UE en la era de los muros: Oportunidades para la Diplomacia Común Europea con Cuba en la etapa post Obama”, en Cornago, De Castro y Moure (eds.) Repensar la Unión Europea: Gobernanza, Seguridad, Mercado Interior y Ciudadanía. Valencia: Editorial Tirant lo Blanch, pp. 585-597. Disponible en: https://editorial.tirant.com/es/libro/ repensar-la-union-europea-gobernanza-seguridad-mercado-interior-y-ciudadania-noe-cornago-9788413362243

Cornago, N. (2011). "El SEAE y la nueva constelación Diplomática Europea”, en Francisco Aldecoa Luzárraga (Coord.), La diplomacia común europea: el servicio europeo de acción exterior. Madrid: Marcial Pons, pp. 83.

Guinea, M. (2011). “El Servicio Europeo de Acción Exterior: Repercusiones para la política de desarrollo europea”, en Francisco Aldecoa Luzárraga (Coord.), "La diplomacia común europea: el servicio europeo de acción exterior". Madrid: Marcial Pons, pp. 118.

Hoffmann, B. (2012). "Más allá de las Relaciones UE-ALC”, en Bases Renovadas para la Relación Unión Europea, América Latina y el Caribe. Fundación EU-LAC. Actas del seminario EU-LAC/GIGA, 17 y 18 de septiembre, Hamburgo, pp. 123-124.

Kennan, G. (2000) "La diplomacia en el mundo moderno”, en J.A. Vásquez (ed.), El pensamiento de los Clásicos. Limusa: Noriega Editores.

Keukeleire, S. et al. (2009). "Reappraising Diplomacy: Structural Diplomacy and the Case of the European Union”. The Hague Journal of Diplomacy, no 4.

Mogherini, F. (2018). La Unión Europea y América Latina: socios en un mundo multilateral. Aula Magna del Colegio "San Gerónimo” de la Universidad de La Habana, pp. 2. Recuperado el 9 de julio de 2019 de www.eeas.europa.eu/sites/eeas/ files/20180103_mogherini_conferencia magistral_uh_cuba.pdf

Moomaw, W. (2007). New Diplomacy. Cambridge: Tufts University.

Ortiz, E. (2016). Unión Europea-Cuba: relación compleja, futuro incierto. Anuario Español de Derecho Internacional. Vol.32. pp. 337-371.

Periódico Granma (2018). "Recibió Díaz-Canel al presidente del Gobierno de España”. Recuperado el 9 de julio de 2019 de: www.granma.cu/cuba/2018-11-22/recibio-diaz-canel-al-presidente-del-gobierno-de-espana-22-11-2018-21-11-29

Periódico Granma (2016). "Reino Unido y Cuba amplían relaciones bilaterales”. Recuperado el 9 de julio de 2019 de: www.granma.cu/mundo/2016-04-28/reino-unido-y-cuba-amplian-relaciones-bilaterales-28-04-2016-22-04-50

Priego, A. (2011). "El Servicio Europeo de Acción Exterior: ¿una revolución para el proceso de integración y para la diplomacia?”. Real Instituto Elcano. Recuperado el 10 de julio de 2019 de: http://www.realinstitutoelcano.org/ wps/portal/rielcano_es/contenido/!ut/p/ a1/04_Sj9CPykssy0xPLMnMz0vMAfGjzOKNQ1zcA73dDQ38 YKNDRwtfN1cnf2cDf1DjfULsh0VAepxmvs!/?WCM 


\section{GLOBAL CONTEXT=/elcano/} Elcano_es/Zonas_es/ARI95-2011

Roig, E. (1959). Los Estados Unidos contra Cuba Libre. Cuba: Editorial Oriente, V I, p. 30

Slater, David, (2004). Geopolitics and the Post-colonial. Rethinking North-South Relations. Oxford: Blackwell Publishing.

Thomas, Isabella, (1996). "La Habana: las implicaciones de Helms-Burton”, Politica Exterior, vol. 53, Nro. 10.

Vadillo, Nerea (2011), "La política exterior de la Unión Europea hacia Cuba (19932003)". Comunicación Social. Sevilla-Zamora. pp. 22

Valdés, Andy. (2016). "Por primera vez en Cuba un canciller del Reino Unido desde 1959" Recuperado el 10 de julio de 2019 de https://www.cubaenmiami.com/ por-primera-vez-en-cuba-un-cancillerdel-reino-unido-desde-1959/

Vargas, José. (2005). “Análisis de Fundamentos de la Teoría Institucional”, Revista Digital Universitaria - UNAM, México, (2-21), Recuperado el 10 de julio de 2019 de http://www.ru.tic.unam.mx:8080/bitstream/handle/123456789/960/ago art84. pdf? sequence $=18$ isAllowed $=y$

Vilariño Pinto, Eduardo. (2000). La Diplomacia Directa. Su alcance y valor actual. Cursos de Derecho Internacional y Relaciones Internacionales de Vitoria-Gasteiz, pp. 293 - 326 Recuperado el 10 de julio de 2019 de https:/www.ehu. eus/documents/10067636/10663089/2000 -Eduardo-Vilarino-Pintos.pdf/745789fbbbdc-5d3b-4847-e756ca0a10b4

\section{Documentos Institucionales}

Decisión del Consejo de 26 de julio de 2010 por la que se establece la organización y el funcionamiento del Servicio Europeo de Acción Exterior (2010/427/UE).

Declaraciones a la prensa (2016) de Federica Mogherini, y Bruno Rodríguez Parrilla. MINREX, Recuperado el 10 de julio de 2019 de www.granma.cu/ mundo/2016-03-11/este-acuerdo-es-unpaso-sin-precedentes-en-la-historiade-los-vinculos-entre-la-union-europea-y-cuba-11-03-2016-23-03-34

Delegación de la UE en Cuba. (2018) Recuperado el 10 de julio de 2019 de www. eeas.europa.eu/delegations/cuba/649/ cuba-y-la-uni\%C3\%B3n-europea_es

Diario Oficial de la Unión Europea, (2010) "Tratado de Funcionamiento de la Unión Europea”. Recuperado el 10 de julio de 2019 de https://www.boe.es/ doue/2010/083/Z00047-00199.pdf

Diario Oficial de la Unión Europea, (2010) “Tratado de la Unión Europea”. Recuperado el 10 de julio del 2019 de https://www. boe.es/doue/2010/083/Z00013-00046.pdf

Diario Oficial de las Comunidades Europeas (1996), "Posición Común de 2 de diciembre de 1996 definida por el Consejo en virtud del artículo J.2 del Tratado de la Unión Europea, sobre Cuba (96/697/PESC)”.

Diario Oficial de la Unión Europea, L 337 I/3 13/12/2016, "Acuerdo de Diálogo Político y de Cooperación UE-Cuba”. Recuperado el 10 de julio de 2019 de www.data. consilium.europa.eu/doc/document/ ST-12504-2016-INIT/en/pdf 
Comisión Europea, (2016) “Estrategia Global para la Política Exterior y de Seguridad de la Unión Europea”.Recuperado el 10 de julio de 2019 de https://eeas.europa. eu/sites/eeas/files/eugs_review_web_0. pdf

\section{Apéndice: Entrevista}

Entrevista al especialista del SEAE vía telefónica en Bruselas-Madrid, 20 de noviembre de 2017.

Entrevista al especialista del SEAE en Santander (España9, el 27 de septiembre de 2016. 